Reprod. Nutr. Dévelop., 1980, 20 (4 B), 1149-1154.

\title{
Influence du nerf vague sur l'activité électromyographique de la région antropylorique chez le Lapin
}

\author{
par Sylvie DELOOF, J. P. ROUSSEAU
}

Laboratoire de Neurophysiologie végétative, CNRS LA n० 308, Université des Sciences ef Techniques de Lille, 59655 Villeneuve d'Ascq Cedex, France

Summary. Influence of the vagus nerve on EMG activity of the antropyloric part of the rabbit stomach.

The electromyographic activity of the stomach antrum and pylorus was recorded in rabbits in acute experimental conditions. Stimulation of the distally cut end of a cervical vagus induced an increase of EMG activity. These results confirm the existence of motor efferent fibers in the vagus. Stimulation of the central end of the vagus inhibited the spontaneous or evoked EMG activity. It is likely that the efferent fibers stimulated by the afferent volley were preganglionic, synapsing with inhibitory myenteric neurons.

\section{Introduction.}

Peu d'études concernent le rôle du nerf vague dans la régulation de la motricité gastrique chez le Lapin (Langley, 1898 ; Burns et Reincke, 1971) ; elles font appel à l'enregistrement glabal de la pression endoluminale. Nous nous proposons de vérifier l'influence vagale sur un autre paramètre, l'activité électromyographique (EMG) de l'antre et du pylore. Déjà décrite chez le Lapin éveillé par Roche et Santini (1970), Ruckebusch, Grivel et Fargeas (1971), puis récemment par l'un d'entre nous (Deloof, 1979), l'activité EMG recueillie par électrodes implantées dans la musculeuse est constituée de salves de potentiels. Dans une série d'expériences préliminaires, nous avons travaillé dans des conditions expérimentales aiguës. Les électrodes à succion d'abord employées recueillent un rythme électrique de base d'ondes lentes, de 4 à 5 par minute, sur lesquelles se greffent des salves de potentiels. Elles ne permettent cependant pas de conserver une telle activité lors des contractions évoquées par la stimulation, qui les déplacent. Aussi, avens-nous étudié l'influence du nerf vague sur l'activité EMG de la région antropylorique, recueillie par des électrodes implantées dans la musculeuse.

\section{Matériel et méthodes.}

Les expériences sont réalisées chez 8 lapins. Nous pratiquons l'ablation du cortex cérébral par aspiration, sous anesthésie induite par le pentobarbital sodique (Nem- 
butal), à raison de $35 \mathrm{mg} / \mathrm{kg}$. Le temps mis pour terminer la décortication permet la dissipation des effets de l'anesthésie. On effectue une injection de curarisant (Flaxe$\mathrm{dil}$ ), à la dose de $1 \mathrm{mg} / \mathrm{kg}$, par la voie intraveineuse. L'animal est ventilé artificiellement pendant toute la suite de l'expérience.

Les extrémités périphérique et centrale d'un nerf vague sectionné en région cervicale sont placées sur des électrodes de stimulation, en crochets de platine. Le tout est recouvert d'huile de paraffine maintenue à $37^{\circ} \mathrm{C}$. Les électrodes sont reliées par l'intermédiaire d'une unité d'isolement Grass à un neurostimulateur Racia à tension de sortie réglable.

Une laparotomie médiane permet de découvrir l'estomac. Les électrodes de réception sont formées de 3 fils de nickel-chrome isolés, dont l'extrémité dénudée est fixée dans la musculeuse de la région antropylorique (Ruckebusch, Grivel et Santini, 1970). Les activités électromyographiques recueillies en enregistrement bipolaire sont visualisées grâce à un enregistreur à plumes, après amplification par un amplificateur dont la bande passante est comprise entre 0 et $300 \mathrm{~Hz}$.

\section{Résultats.}

L'activité spontanée est constituée de salves de potentiels, dont la fréquence varie de 1 à 3 par minute. Toutefois, sur 8 expériences réalisées, elle n'est enregistrée que dans 2 cas.

\section{1. - Effet de la stimulation des fibres vagales efférentes.}

En l'absence d'activité spontanée, nous stimulons l'extrémité périphérique d'un nerf vague cervical sectionné, par des chocs de $1 \mathrm{~ms}$ de durée, à la fréquence de 4 par seconde. Pour une tension de $9 \mathrm{~V}$, des salves de potentiels de 3 à $10 \mathrm{~s}$ de durée apparaissent sur l'antre et le pylore avec une latence de 5 à $10 \mathrm{~s}$, à la fréquence de 3 par minute (fig. 1A). Elles comprennent 3 à 15 polentiels dont l'amplitude varie entre $0,1 \mathrm{mV}$ et $1 \mathrm{mV}$. L'arrêt de la stimulation entraîne celui des activités provoquées. Les électrodes situées à plus de $4 \mathrm{~cm}$ en amont du pylcre ou sur le corpus ne recueillent pas d'activité lors de la stimulation vagale efférente. Les effets de la stimulation centrifuge sont indépendants du vague stimulé ef de la durée des chocs imposés. Par contre, pour une durée et une tension constantes des chocs, la réponse varie selon la fréquence de stimulation. Elle apparaît pour des fréquences supérieures à 1 ou 2 chocs par seconde. Elle est maximale pour des fréquences de 4 à 12 chocs par seconde et diminue lorsqu'on augmente la fréquence de stimulation au-delà de 12 chocs par seconde.

Lorsqu'on enregistre une activité spontanée, la stimulation des fibres efférentes entraîne son augmentation : la durée des salves est doublée, de 5 à $10 \mathrm{~s}$, alors que leur fréquence n'augmente que de 3 à 3,5 par minute. Le nombre, l'amplitude et la fréquence des potentiels sont nettement accrus. L'activation cesse dès la fin de la stimulation et les salves ne réapparaissent qu'une minute après (fig. 1B). Tout se passe comme si les effets facilitateurs de la stimulation étaient suivis d'une inhibition du rythme, de courte durée. 
Ant.

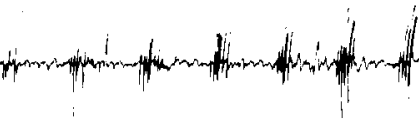

Pyl.

B

Ant.

Ant.

Pyl.

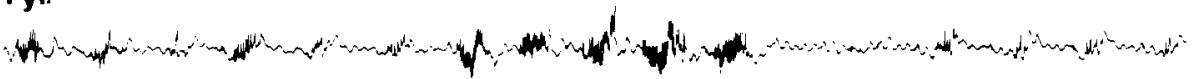

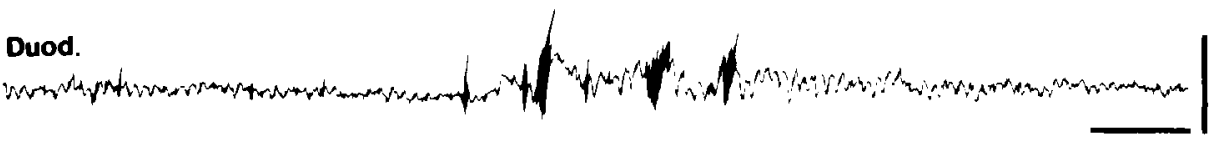

FIG. 1. - Effets de la stimulation des efférences vagales sur l'activité EMG de l'antre (Ant.), du pylore (Pyl.) ef du duodénum (Duod.). La stimulation itérative de l'extrémité périphérique du vague provoque l'apparition de salves de potentiels en $A$ et détermine l'augmentation de l'activité spontanée en B. Etalonnage : amplitude : $1,5 \mathrm{mV}$; temps : $30 \mathrm{~s}$.

2. - Effets de la stimulation des fibres vagales afférentes.

A. - Effets sur l'activité EMG spontanée. - L'extrémité centrale d'un vague sectionné est stimulée par des chocs de 12 à $25 \mathrm{~V}$, de $1 \mathrm{~ms}$ de durée, délivrés à la fréquence de 5 par seconde. On observe pendant toute la durée de la stimulation, l'inhibition de l'activité spontanée de l'antre et du pylore (fig. 2A). Moins de 10 s après l'arrêt de la stimulation, les salves antrales et pyloriques réapparaissent à la fréquence de 3 par minute, supérieure à la fréquence initiale. Les potentiels sont plus nombreux que ceux des salves précédant la stimulation. Cet effet de rebond se poursuit pendant plusieurs minutes. L'inhibition réflexe manque lorsque le vague contralatéral est préalablement sectionné.

B. - Effets sur l'activité EMG évoquée par la stimulation des efférences vagales. L'arrêł de l'activité antropylorique observé dans l'expérience précédente pourrait être interprété comme une interruption normale et aléatoire de l'activité en salves et ne résulterait pas de la stimulation des afférences vagales. Pour vérifier ce point, nous évoquons un rythme de salves en stimulant l'extrémité périphérique du vague sectionné, sur les préparations dont l'estomac ne présente pas d'activité spontanée. La stimulation de l'extrémité centrale entraîne dans 65 p. 100 des cas l'inhibition des 
activités évoquées, quelles que soient la durée et la fréquence des chocs délivrés. Lorsqu'elle dure une minute, son arrêt est immédiatement suivi de la réapparition des activités évoquées qui ne présentent pas de phénomène de rebond (fig. $2 \mathrm{~B}$ ). Si la stimulation centrale persiste plusieurs minutes, l'inhibition est d'abord totale, puis on observe un effet d'échappement: a reprise des activités évoquées s'effectue avant l'arrêt de la stimulation centrale.

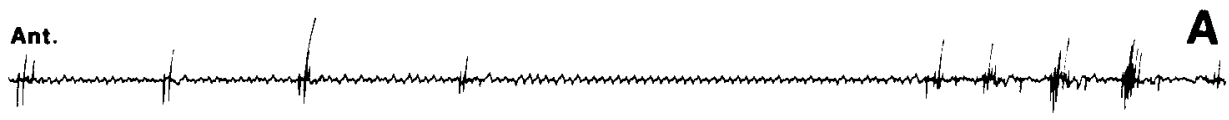

Pyl.

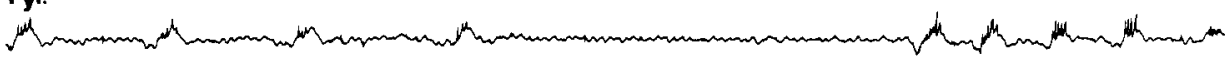

Ant.1

Ant.2

Pyl. and

FIG. 2. - Effets de la stimulation des afférences vagales sur les activités EMG de l'antre (Ant.) ef du pylore (Pyl.). En A, la stimulation itérative de l'extrémité centrale du vague défermine l'inhibition de l'activité spontanée. On observe l'effet de rebond à l'arrêt de la stimulation. En B, la stimulation centrale provoque l'inhibition de l'activité antropylorique évoquée par la stimulation périphérique. Les lignes en traits pleins et en tirets correspondent aux durées respectives des stimulations périphérique et centrale. Etalonnage : amplifude : $1,5 \mathrm{mV}$; temps : $30 \mathrm{~s}$.

\section{Discussion.}

1. - Effets moteurs. - La stimulation de l'extrémité périphérique du vague sectionné permet de retrouver les effets excitateurs classiques des efférences vagales sur la motricité gastrique. Depuis les expériences de Langley (1898), les travaux sont nombreux qui montrent l'existence, dans le nerf vague, de fibres motrices à destination de l'estomac des monogastriques comme des polygastriques. Chez l'animal éveillé (Mouton, Chien), la technique des sułures nerveuses hétérogènes croisées vago-phréniques permet de recueillir des activités qui traduisent la mise en jeu de neurones préganglionnaires vagaux, moteurs de l'estomac (Dussardier, 1960 ; Miolan ef Roman, 1978). A la différence du Mouton où le rythme gastrique est élaboré par des décharges rythmiques des centres moteurs bulbaires (Dussardier, 1960), nous pensons que le vague moteur ne joue, chez le Lapin, qu'un rôle modulateur d'une motricité intrinsèque. Effectivement, la bivagotomie ne supprime pas le rythme antropylorique, qui reste déprimé seulement les trois premiers jours après la section (Deloof, 1979). 
2. - Effets inhibiteurs. - Dans les deux cas où nous enregistrons une activité spontanée, nous n'observons pas d'inhibition de la motricité antropylorique par la stimulation des efférences vagales quels qu'en soient les paramètres. On pourrait penser que dans nos expériences de stimulation réflexe, l'arrêł de la motricité est la conséquence de l'inhibition centrale par les afférences d'une voie efférente facilitatrice. Mais cette interprétation n'explique pas les résultats obtenus sur les estomacs dont l'activité est évoquée par la stimulation du vague périphérique. L'inhibition ne peut résulter dans ce cas que d'une action périphérique du vague : la volée afférente stimulerait des neurones préganglionnaires vagaux faisant synapse au sein des plexus de la région antropylorique, avec des neurones inhibiteurs. Il est remarquable d'observer dans nos expériences l'effet d'échappement lorsque cette stimulation est de plus longue durée. Or un fait caractéristique des réponses inhibitrices de l'intestin à la stimulation transpariétale des neurones non cholinergiques non adrénergiques est effectivement l'excitation de rebond qui suit le plus souvent l'arrêt de la stimulation, ou la reprise de l'activité au cours d'une période de stimulation itérative (Burnstock, 1979).

On sait que des fibres vagales, à seuil d'excitation élevé, constituent la voie efférente d'ún réflexe vago-vagal responsable du relâchement de la musculature gastrique (Martinson, 1964 ; Burns et Reincke, 1971). Ce sont vraisemblablement celles dont Miolan et Roman (1974) enregistrent l'activité spontanée chez le Chien éveillé, pendant la relaxation réceptive de l'estomac.

Les fibres vagales inhibant la motricité antropylorique ont certainement un rôle physiologique différent. Le rythme antropylorique est d'ailleurs augmenté pendant le repas (Deloof, 1979). Elles constifuent vraisemblablement la voie effectrice des réflexes duodéno-gastriques inhibiteurs de la motricité, qui interviennent dans la régulation de la vidange de l'estomac.

En résumé, nous enregistrons l'activité électromyographique de l'antre ef du pylore, chez le Lapin, dans des conditions expérimentales aiguës. Nous montrons l'existence dans le vague, à côté d'un contingent de fibres motrices, de fibres mises en jeu par voie réflexe et responsables de l'inhibition de la motricité antropylorique. Ces fibres vagales attaquent vraisemblablement les neurones post-ganglionnaires inhibiteurs des plexus de la région antro-pylorique.

Journées Ingestion-Digestion-Absorption de l'Association française de Nutrition, Paris, $15-16$ novembre 1979.

\section{Références}

BURNS T. D., REINCKE D. A., 1971. Gastric motility inhibition and excitation elicited by vagal stimulation. Amer. J. Physiol., 221, 226-229.

BURNSTOCK G., 1972. Purinergic nerves. Pharmacol. Rev., 24, 509-581.

BURNSTOCK G., 1979. Autonomic innervation and transmission. Brit. med. Bull., 35, $256-262$.

DELOOF S., 1979. La motricité antropylorique chez le Lapin. Rôle de l'innervation vagale. Th. $3^{\mathrm{e}} \mathrm{cycle}$, Lille, 111 PP.

DUSSARDIER M., 1960. Recherches sur le confrôle bulbaire de la molricité gastrique chez les Ruminanis. Th. Doct. ès Sci., Paris, 196 Pp. 
LANGLEY J. N., 1898. On inhibitory fibres in the vagus for the end of the œsophagus and the stomach. J. Physiol. Lond., 23, 407-414.

MARTINSON J., 1964. The effect of graded stimulation of efferent vagal nerve fibres on gastric motility. Acta physiol. scand., 62, 256-262.

MIOLAN J. P., ROMAN C., 1974. Décharge unitaire des fibres vagales efférentes lors de la relaxation réceptive de l'estomac du chien. J. Physiol. Paris, 68, 693-704.

MIOLAN J. P., ROMAN C., 1978 . Discharge of efferent vagal fibers supplying gastric antrum : indirect study by nerve suture technique. Am. J. Physiol., 235, E366-E373.

ROCHE M., SANTINI R., 1970. Etude électrophysiologique de la motricité gastrique. I. Electrogastromyographie comparée. C. R. Soc. Biol., 164, 1528-1534.

RUCKEBUSCH, Y., GRIVEL M. L., FARGEAS M. J., 1971. Activité électrique de l'intestin et prise de nourriture conditionnelle chez le lapin. Physiol. Behav., 6, 359-365.

RUCKEBUSCH Y., GRIVEL M. L., SANTINI R., 1970. Technique de dérivation chronique de l'activité électrique et mécanique du tractus digestif chez l'animal éveillé. Soc. Sci. vet. Méd. comp., 337-346. 